\title{
A Consideration on Visual Strategy of Fovea and Saccadic Movement from Experimental Results
}

\author{
Yoshiaki Ajioka \\ Department of Information and Control Engineering, Toyota Technological Institute \\ 2-12-1 Hisakata, Tempaku-ku, Nagoya 468 Japan
}

Presently, my most interesting aspect on human visual perception was a visual strategy of fovea and eye movement, particularly saccadic movement, because Adaptive Junction, which is a continuoustime asymmetric neural network, has suggested that some kinds of optical illusion can be easily explained in terms of the visual strategy, by simulating duck-rabbit illusion and interblocking. In this work, then, an experimentation that more than one hundred subjects see either a duck-rabbit figure or some similar figures was executed for verifying the suggestion.

I briefly explain here about this experimental method using a questionnaire survey.

1. Four figures such as a duck-rabbit figure, a figure except an eye, a figure except rabbit's nose, a figure except duck's mouth were prepared.

2. These figures are magnified within the size of $8 \mathrm{~cm}$ by $8 \mathrm{~cm}$.

3. The questionnaire consists of three papers representing directions, two questions and one of the above figures.

4. First question requires to answer what the figure shows at a glance (within one second).

5. Second question requires to answer what the figure shows after watching it (for more than twenty seconds).

6. Experimental subjects can take and move these questionnaire papers freely.

Now, from some experimental results, three figures except a feature seem not to make many experimental subjects occur duck-rabbit illusion. Let's compare these results with one another.

First, although the figure except an eye roughly represents the contour of an object, almost all subjects answer a bird at a glance and more than fifty percents of subjects also answer a bird after watching. Under consideration of the effect of contour, the duck-rabbit figure trends to be recognized as a bird. It is not difficult for us to guess that this trend is very remarkable in the case of a glance. Next, an eye of the duck-rabbit figure seems to excite rabbit's nose because many subjects can not find out the nose despite that the figure except the eye includes it, and furthermore all subjects can not recognize the figure except rabbit's nose as a rabbit. In short, the subjects can not recognize a rabbit without rabbit's nose.

By the way, I am afraid many subjects occurred duck-rabbit illusion while watching the figure except duck's mouth. If the removed area was so large as it hid either one of duck's bills, many experimental subjects would answer only a rabbit. However, only the result of the figure except duck's mouth indicates that more than fifty percents of experimental subjects can recognize the figure as a rabbit at a glance. This result suggests not only that duck's mouth is important for recognition of a duck but also that the eye in the center of the figure excites two neighbor features, duck's mouth and rabbit's nose. In brief, the nearer two features are with each other, the interaction of them is stronger, but a direction from one feature to another seems not concerned with recognition. If a human visual perception system had all possible neurons for each combination of neighbor features, it would need the astronomical figures of neurons and synapses. Thus, it seems that saccadic movement avoids this problem and furthermore the next feature from the eye decides a first recognition result of duck-rabbit illusion.

Concluding this work, these experimental results support the idea that duck-rabbit illusion is caused by the visual strategy of fovea and saccadic movement. This idea certainly shows a body plays an important role for cognitive behavior. 